\title{
BOOKS REVIEW
}

\section{Hans Wiesmeth}

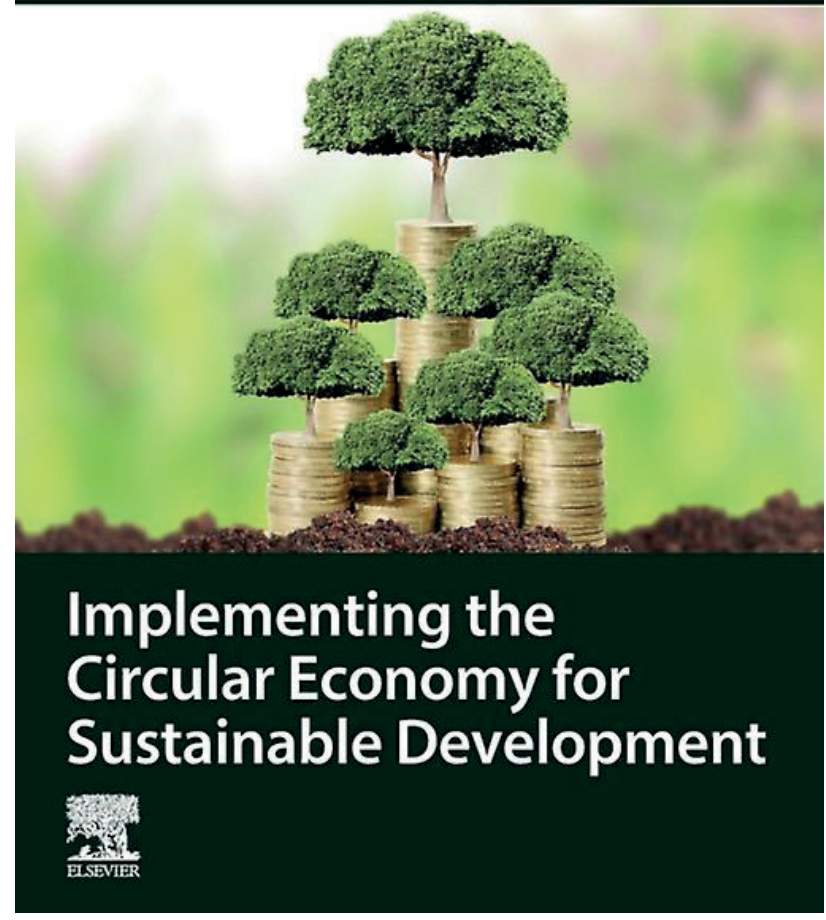

\section{IMPLEMENTING THE CIRCULAR ECONOMY FOR SUSTAINABLE DEVELOPMENT}

\section{Author: Hans Wiesmeth}

Circular economy (CE) is a mainstream concept, yet a quick glance is enough to understand that its definition and application is heterogenous, and highly context dependent. What is the difference between the economy as we know it and the circular economy? And what are its boundaries? The book "Implementing the circular economy for sustainable development" addresses these questions and beyond, giving a comprehensive and detailed overview both on the theory and on practical applications of $\mathrm{CE}$, thus supporting those who approach the topic for the first time and those wishing to acquire in-depth knowledge.

Section one of the book starts from definitions and meaning attributed to "circular economy" (chapter 1), by analyzing different policy frameworks (chapter 2), then showing how the concept is represented in the scientific literature (chapter 3 ) and detecting the leaders and the followers countries in the adoption of CE agendas. Is there a unique definition of circular economy in practice or in scientific literature? [spoiler alert: no, there is not!]. And what about business models? Are they profitable and scalable? These are some of the questions addressed in the first three chapters. Chapter 4 describes the possible drivers that lead a country to undertake a rigorous policy strategy towards a circular economy. According to the author, the country characteristics influencing such path are a) Endowment with natural resources b) Density of the population, respectively land available for landfilling c) Level of environmental awareness d) Motivation for strategic behavior. Some countries have been analyzed as early adopters (leaders), such as Germany with its advanced legislation about waste; and China with its pioneering development strategy implemented already in 2002. Late adopters (followers) have been identified in Georgia and Russia, while USA have been described as laying "in between". Chapter 5 presents environmental laws, directives and ordinances from different countries targeting crucial topics of a circular economy, such as waste management and climate change regulations in the European Union (EU), or the case of the "California's bottle bill".

In the second section of the book, the nexus between the economy and the environment is explored. The concept of environmental commodity is presented (Chapter 6), and basic concepts as perceived scarcity and perceived feedback from environmentally friendly actions are illustrated. Chapter 7 focuses on the allocation of environmental commodities. According to the author, the Tragedy of the Commons and the Prisoners' Dilemma are two mechanisms able to explain the failure or success of developing an environmental strategy; indeed, the case study of an involuntary policy failure is shown through the example of the German refillable quota issue, explained through the prisoners' dilemma. Chapter 8 presents an introduction to behavioral economics, bringing to the forefront some principles of possible relevance for environmental economics and for the implementation of circular economy initiatives. Prospect theory, limited information, the role of social norms and finally, the environmental awareness and perceived feedback, all play a role in the possible success of the CE implementation. Once again, a case study clarifies the issue, but it's in paragraph 8.5 where we find the ultimate answer to the question: how useful are behavioral economics to implement a circular economy?

Section three of the book analyzes the role of technology in boosting the CE implementation. Innovative technologies are crucial in enabling the development of CE in practice, but their adoption also entails some drawbacks. Yet, where are the roots of the idea that technology can help achieve a circular economy? Chapter 10 conceptualizes the link between CE and the technological endeavor re- 
lying on the industrial ecology theory. After that, it provides a frame about the environmental technologies, their economy and role in the global markets, as well as their interconnectedness with policy. Chapter 11 enquires into the role of information (and its asymmetries), its links with governmental policies to favor the adoption of CE measures and it brings a case study of (partial) success: the promotion of renewable energy sources in Germany. Chapter 14 explains the rebound effects and path dependencies (technological, societal) and illustrates their possible impact on the implementation of a circular economy. Chapter 15 examines how the digital economy and the circular economy boost each other and sometimes "impede each other in a variety of ways": the most practical case of half success is represented by smart cities (13.1.1), while some challenges are illustrated by the case of e-commerce and online shopping.

Section four addresses the issues of policies to implement environmental measures. It starts from the description of some policy regimes (chapter 14) such as laissez-faire, technology-guidance, command-and-control policies, augmented market system and it ends with the importance of engaging stakeholders through appropriate framework conditions. Chapter 15 examines environmental standards and the mechanism through they are changed. The case study reports on the emission standards for vehicles. Chapter 16 deals with market-oriented policy tools, explaining the main set of measures commonly applied: pollution tax- with a focus on the polluter pays principle; tradable emission certificates and related markets; last, a brief examination of the Coase Theorem and some behavioral aspects relating to voluntary contributions and flexible information-based policies are illustrated. Eventually, chapter 17 considers holistic policy approaches with their origins, their economic background, and practical experiences gained. The holistic approach takes into account a large set of stakeholders, information issues and need for inter-disciplinary target-setting. Two practical cases are carefully illustrated: the Integrated Waste Management policies and the Extended Producer Responsibility. Chapter 18 analyses the economics of waste hierarchy, highlighting the failure of the waste prevention (defined as "the forgotten child" of the waste management) due to societal path dependencies. The issue of recycling and its path dependencies is illustrated as well.

Section five offers a guidance to implement holistic policies, defined as "integrated Environmental Policies (IEP)". In chapter 19, the constitutive elements of an IEP are analyzed with reference to the question "where are we on the road to a circular economy?". Germany's case about the state of the art of the CE implementation is reported. Chapter 20 is focused on packaging waste, while chapter 21 is about Waste Electrical and Electronic Equipment (WWEE) and End-of-life vehicles (ELV). Chapter 22 faces the topic of climate change mitigation with special attention paid to the emissions, from the Kyoto protocol to Copenhagen, Paris and Madrid. An explanation about the Emission Trading System (ETS) and its application at national and global level follows. Chapter 23 is about plastics and its potential reduction thanks to an IEP for plastic waste; the chapter illustrates the different type of plastics, the critical issues about recycling, the international market and China's ban and it ends with the constitutive elements of an IEP on plastic. Chapter 24 is about textiles waste, and it follows a similar structure of the previous chapter. The last chapter (25) presents conclusive remarks about a real possibility for the system to change, along with a summary of the issues presented in the book, connected to the potential development of specific IEPs. Some reflections on the impact of the lockdown on the system and the potentialities shown to change it are offered.

Overall, this book examines all the aspects that can determine the success or failure of the CE application; the theoretical discussion is complemented with the analysis of both specific IEP pillars and single case studies.

Anybody who wants to learn about circular economy in theory and practice may benefit from it, as the content is highly technical, but the language is easy-going. The book is recommended for professionals, consultants, policy makers and researchers. Master students may benefit from it as well.

\section{Claudia Giordano}

Junior Assistant Professor (RTDa), University of Bologna (IT) e-mail: claudia.giordano4@unibo.it

\section{ABOUT THE AUTHOR Hans Wiesmeth}

Dr. Hans Wiesmeth is the President of the Saxon Academy of Sciences and Humanities, Leipzig, Germany, and a professor emeritus of the Faculty of Business and Economics of Technical University of Dresden, Dresden, Germany. $\mathrm{He}$ also works as the Academic Director of the Laboratory for International and Regional Economics at Ural Federal University, Yekaterinburg, Russia. Dr. Wiesmeth has visited many international universities, including the University of Western Ontario, London, Canada, and York University, Toronto, Canada. He has participated in various national and international research projects, including EU projects on environmental issues such as waste management and renewable energy sources in developing countries.

\section{Book Info:}

Editors: Hans Wiesmeth Imprint: Elsevier

Year of publication: 2020

Page Count: 326

Paperback ISBN: 9780128217986 